\title{
Nota
}

\section{Ampliación del área de distribución de Micrurus camilae (Serpentes: Elapidae) en el Caribe colombiano}

\section{Range extension of Micrurus camilae (Serpentes: Elapidae) in the Colombian Caribbean}

\author{
Sergio Alejandro Peláez Plazas ${ }^{(1)}$, Lilia Alejandra Perlaza Berrío
}

\section{Resumen}

Se presenta el primer registro de la especie Micrurus camilae para el departamento de Sucre, Colombia, ampliando su área de distribución y contribuyendo con datos sobre su hábitat. El individuo se encontró en un parche de vegetación en bosque seco tropical, a diferencia de los reportes anteriores donde se localiza en bosque húmedo tropical.

Palabras clave. Bosque seco tropical. Bosque húmedo tropical. Elapidae. Proteroglifa. Rango de Distribución.

\begin{abstract}
We present the first record of the snake Micrurus camilae for the department of Sucre, Colombia, extending its known distribution area and contributing with data about its habitat. The specimen was found in a tropical dry forest patch, in contrast with previous records, in which $M$. camilae was located in tropical rainforests.
\end{abstract}

Keywords. Distributional Range. Elapidae. Proteroglyphous. Tropical dry forest. Tropical rainforest.

\section{Introducción}

El género Micrurus (Elapidae) comprende aproximadamente 31 especies en Colombia, siendo uno de los grupos de serpientes más diversos en el país (Uetz et al., 2019). Estas serpientes se distribuyen principalmente en bosques húmedos y secos y en bosques premontanos (Pitalua et al., 2018). Los individuos de este género presentan hábitos fosoriales, crepusculares, y una dentición proteroglifa que le permite suministrar un potente veneno neurotóxico (Pitalua et al., 2018).

Micrurus camilae es endémica de Colombia. En su descripción original se señala que la especie habita en el bosque húmedo tropical del municipio de Tierralta, Córdoba (Renjifo \& Lundberg, 2003). Posteriormente, se realizaron tres registros de la especie en los municipios de Valencia (departamento de Córdoba), La Lizama y El Cedral (departamento de Santander) y Tarazá (departamento de Antioquia; Alzate, 2014; MenesesPelayo \& Caballero, 2019; Ines Hladki et al., 2016). Hasta el momento para esta especie no se han reportado datos ecológicos o su estado de conservación, debido a que la información actual es deficiente (Alzate, 2014; Ines Hladki et al., 2016). Sin embargo, con estos reportes se ha concluido que la especie habita en zonas con alto grado de fragmentación e intervención antrópica (Meneses-Pelayo \& Caballero, 2019). 
Micrurus camilae se caracteriza por presentar un patrón de coloración bicolor de anillos negros y amarillos, con una franja ancha mediodorsal de color rojo a lo largo de todo el cuerpo (Renjifo \& Lundberg, 2003). El primer anillo se inicia sobre la región occipital, cubriendo las escamas dorsales. Presenta de 30 a 35 anillos amarillos (de 3 a 7 escamas de ancho) alternados con 31 a 36 anillos negros (de 4 a 8 escamas de ancho), cola con 4 anillos negros, 4 amarillos y escama anal dividida (Renjifo \& Lundberg, 2003). Este estudio presenta el primer registro de la especie Micrurus camilae para el departamento de Sucre, Colombia, extendiendo hacia el norte su rango de distribución y contribuyendo con datos sobre su hábitat.

\section{Materiales y métodos}

En enero de 2017 (época seca), se encontró un individuo de Micrurus camilae en un parche de vegetación herbácea y hojarasca cerca de la zona que limita con la hacienda Pajarito, en el municipio de Colosó, Sucre

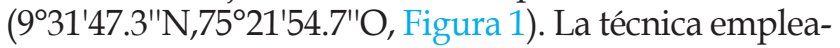
da para el registro del espécimen fue inspección por encuentro visual (VES) y la determinación del individuo siguió la descripción de Renjifo \& Lundberg (2003). El ejemplar no fue recolectado; solo se obtuvieron registros fotográficos.

\section{Resultados}

El individuo de Micrurus camilae se observó reposando sobre vegetación herbácea y hojarasca en horas de la mañana (alrededor de las 9:00 am) cerca de la quebrada Charco Azul, a una altura de 145 m s.n.m.

El patrón de coloración del cuerpo es similar al descrito por Renjifo \& Lundberg (2003): 34 anillos negros que cubren de 4 a 7 escamas dorsales de ancho alternados con 34 amarillos de 4 a 5 escamas de ancho; 4 anillos negros, 4 amarillos en la cola y escama anal dividida (Figura 2). No obstante, el patrón de coloración de la cabeza se asemeja más a la descripción del individuo encontrado por Meneses-Pelayo \& Caballero (2019) en el valle medio del río Magdalena, al presentar dos anillos negros discontinuos, el primero cubriendo la escama rostral, y el segundo cubriendo las escamas frontal, supraocular, preocular, postocular y parte anterior de las escamas parietales; las escamas internasales y prefrontales son de color rojo, mientras que Renjifo
\& Lundberg (2003) describen la coloración de la cabeza como totalmente negra (desde la rostral hasta las supraoculares, incluyendo la frontal y la parte posterior de la parietal). Sin embargo, la disposición de las escamas se ajusta a la descripción de los autores, siendo la escama rostral más ancha que alta y visible dorsalmente, dos internasales más largas que anchas, escama frontal y dos escamas parietales más largas que ancha, escama loreal ausente, nasales divididas y una preoculas más larga que ancha (Renjifo \& Lundberg, 2003).

\section{Discusión}

En los trabajos realizados en el bosque seco tropical de los montes de María (Sucre, Colombia) se han registrado 33 especies de serpientes (Galván et al., 2009; Pineda et al., 2015), de las cuales se reconocen dos de elápidos: Micrurus dissoleucus y Micrurus sp. (Galván et al., 2009). Sin embargo, no existe una caracterización morfológica de Micrurus sp. para poder compararla con las demás especies de elápidos de la zona, incluyendo a M. camilae. En otros trabajos se compara esa especie con $M$. dissoleucus y M. dumerilii, mostrando únicamente que las tres especies son ovíparas y que difieren notablemente en el patrón de coloración (Vargas et al., 2019).

Trabajos previos (Alzate, 2014; Ines Hladki et al., 2016; Meneses-Pelayo \& Caballero, 2019; Renjifo \& Lundberg, 2003), han señalado que $M$. camilae se encuentra en bosque húmedo tropical, entre 88 y 176 m s.n.m. En este estudio, M. camilae se encontró en un fragmento de bosque seco tropical a una altura de 145 m s.n.m., 190 $\mathrm{km}$ al noreste de la localidad más septentrional conocida hasta ahora para la especie. Es necesario ampliar los estudios sobre $M$. camilae para conocer su densidad poblacional y estado de amenaza, con el fin de promover su conservación y protección.

\section{Agradecimientos}

Agradecemos a la Corporación Autónoma Regional de Sucre (CARSucre) por brindarnos la oportunidad ingresar a la estación Primatológica de Colosó; a la Universidad Distrital Francisco José de Caldas por su apoyo durante esta investigación; al señor Pedro Márquez por su paciencia y compañía en algunos de nuestros muestreos; y al profesor Oscar Javier Mahecha por su apoyo y dirección en este proyecto. 

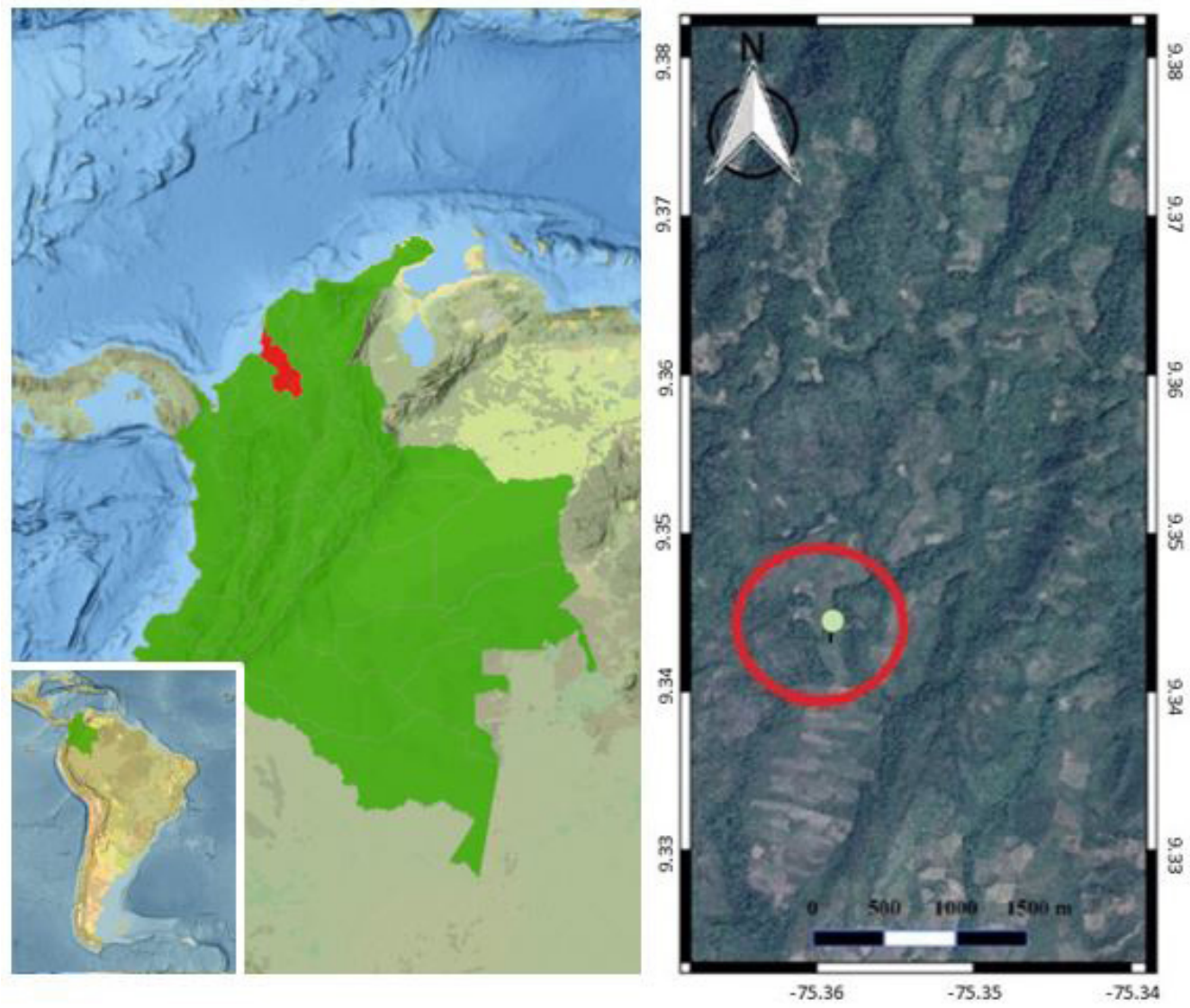

Figura 1. Localización del registro de Micrurus camilae en el municipio de Colosó, departamento de Sucre, Colombia.
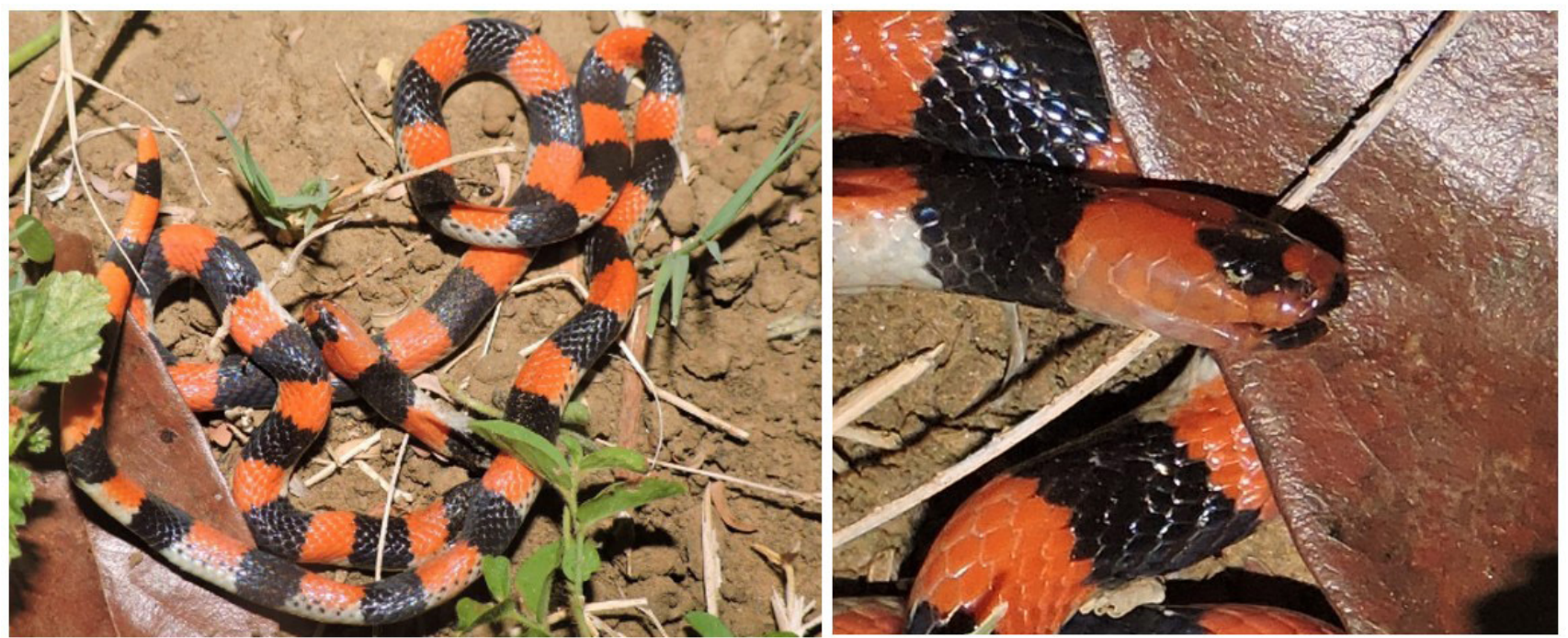

Figura 2. Individuo de Micrurus camilae en Colosó, Sucre, Colombia. 


\section{Referencias}

Alzate, E. (2014). Geographic distribution: Micrurus camilae. Herpetological Review, 45(2), 285-286.

Galván, S., Sierra, M., Gómez, F., De La Ossa, V. \& Fajardo, A. (2009). Biodiversidad en el área de influencia de la Estación Primates de Colosó, Sucre, Colombia. Revista Colombiana Ciencia Animal, 1(1), 98121. https:// doi.org/10.24188/recia.v1.n1.2009.417

González, C., Cabrera, O., Munstermann, L. \& Ferro, C. (2006). Distribución de los vectores de Leishmania infantum (Kinetoplastida: Trypanosomatidae) en Colombia. Biomedica, 26(1), 3-4.

https://doi.org/10.7705/biomedica.v26i1.1501

Ines Hladki, A., Ramírez, M., Renjifo , J. \& Urbina, N. (2016). Micrurus camilae. The IUCN Red List of Threatened Species 2016: e.T44581946A44581949. http://dx.doi.org/10.2305/IUCN.UK.2016-1.RLTS.

Lehtinen, R., Ramanamanjato, J. \& Raveloarison, J. (2003). Edge effects and extinction proneness in a herpetofauna from Madagascar. Biodiversity and Conservation, 12(7), 1357-1370.

https:/ / doi.org/10.1023/A:1023673301850

Meneses-Pelayo, E. \& Caballero, D. (2019). New records and an updated map of distribution of Micrurus camilae Renjifo \& Lundberg, 2003 (Elapidae) for Colombia. Check List, 5(3), 465-469.

https:// doi.org/10.15560/15.3.465

Molina, C. Señaris, J., Lampo M. \& Rial. A. (eds.). (2009). Introducción a los anfibios de Venezuela: Estado del conocimiento y recomendaciones para su conservación. (Informe técnico). Caracas, Venezuela: Universidad Central de Venezuela, Conservación Internacional Venezuela, Instituto de Zoología y Ecología Tropical. 23 pp.

Mueses, V. (2011). Conservación de la biodiversidad o desarrollo social: una deliberación bioética (Tesis de maestría). Bogotá D. C.: Pontificia Universidad Javeriana, Instituto de Bioética. 37 pp.

Pineda, A., Jiménez, S., Fernández, C., Peña, L., Granda, H., Morelo, L., Vela, I. \& González, J. (2015). Planeación ambiental para la conservación de la biodiversidad en las áreas operativas de EcoPetrol: Ventana Montes de Maria, Colosó, Sucre. (Informe Técnico). Bogotá D.C.: Instituto de Investigación de Recursos Biológicos Alexander von Humboldt. 30 pp.

Pitalua, Y., Rengifo J. \& Rivas, L. (2018). Aportes a la distribución del género Micrurus (Serpentes: Elapidae) en el Departamento del Chocó, Colombia. Revista Colombiana Ciencia Animal, 10(2), 131-142. https:// doi.org/10.24188/recia.v10.n2.2018.649

Renjifo, J. \& Lundberg M. (2003). Una especie nueva de serpiente coral (Elapidae, Micrurus), de la región de Urrá, municipio de Tierra Alta, Córdoba, Noroccidente de Colombia. Academia Colombiana de Ciencias Exactas, Físicas y Naturales, 27(102), 142-144.

Uetz, P., Freed, P. \& Hosek, J. (eds). (2019). The Reptile Database. Retrieved from http:/ / www.reptile-database.org

Vargas, F., Muñoz, J. \& Morales, M. (Coord.) (2019). Biología de los anfibios y reptiles en el bosque seco tropical del norte de Colombia. Tunja, Colombia: Editorial UPTC. 
Sergio Alejandro Peláez Plazas

Universidad Distrital Francisco José de Caldas

Bogotá, Colombia

sergi.alejopelaez@gmail.com

https://orcid.org/0000-0002-8357-2199

\section{Lilia Alejandra Perlaza Berrío}

Universidad Distrital Francisco José de Caldas

Bogotá, Colombia

li.alejandraperlazaberrio@gmail.com

https://orcid.org/0000-0002-0138-1242

\section{Ampliación del área de distribución de Micrurus} camilae (Serpentes: Elapidae) en el Caribe colombiano

Citación del artículo: Peláez, S.A. \& Perlaza, L.A. (2020). Ampliación del área de distribución de Micrurus camilae (Serpentes: Elapidae) en el Caribe colombiano. Biota Colombiana, 21(1), 104-108. DOI: 10.21068/ c2020.v21n01a07

Recibido: 31 de octubre de 2018

Aprobado: 2 de diciembre de 2019

108 | Biota Colombiana 21 (1) - 2020 